\title{
Investigation of the change of the shrinkage properties in contradiction to the change of the composition of cotton polyester spandex denim fabrics
}

\begin{abstract}
The aim of this research is to investigate the shrinkage properties of cotton polyester spandex denim fabrics of different fiber content. Cotton fabrics with spandex have a tendency to shrink when it comes in contact of water. Cotton spandex shrinks more when it comes in contact of hot water. Cotton is swelled up in water; thereby increase the width of fibers, as a result there is the decrease of length. If the cotton composition with spandex increases, the shrinkage property of fabric also increases. The higher the percentage of spandex content in a fabric is, the higher the value of shrinkage is. On the other hand, polyester does not shrink when it comes in contact of water, as manmade polyester fiber does not swell up in water. Three specimen of cotton polyester spandex denim fabrics of different fiber content were used in this research. Finished fabrics were collected from fabric mills for conducting the required shrinkage test with the standard specified by AATCC Test Method 135. This research is practice based and the discoveries are advantageous to the textile professionals. This research proved that the properties of shrinkage depends on the fiber content of cotton polyester spandex denim fabrics and it showed a suitable way for the scholars to further study in this field.
\end{abstract}

Keywords: Cotton polyester spandex, Shrinkage properties, Denim fabrics, Fiber content, Swelling property, Absorbency, Resistance to water, Breathability, perameability
Volume 5 Issue 3 - 2019

\author{
Shariful Islam,' Nishat Tasnim, ${ }^{2}$ Tanvirul Islam ${ }^{3}$ \\ Faridul Islam ${ }^{4}$ \\ 'Department of Fabric Engineering, Bangladesh University of \\ Textiles, Dhaka, Bangladesh \\ ${ }^{2}$ Department of Yarn Engineering, Bangladesh University of \\ Textiles, Dhaka, Bangladesh \\ ${ }^{3}$ Department of Fabric Manufacturing Engineering, Khulna \\ University of Engineering \& Technology, Khulna, Bangladesh \\ ${ }^{4}$ Faridul Islam, Department of Textile Engineering, Faculty of \\ Science and Engineering, City University, Bangladesh
}

Correspondence: Shariful Islam, Department of Fabric Engineering, Bangladesh University of Textiles, Tejgoan, Dhaka-I 208, Bangladesh, Tel +880 I 9 | I509933,

Email Sharifultextiles@gmail.com

Received: December 20, 2018 | Published: June 12, 2019

\section{Introduction}

This research has a great significance in clothing engineering since controlling of shrinkage is a vital issue now a day for the stretched garments. Trials should be carried out to reduce the problems of shrinkage. Shrinkage has some negative impact upon wearer satisfaction while washing.

Different academics and intellectuals worked interrelated to this research at different times and they established different concept. Sometimes their perception was similar and sometimes their perception was widely dissimilar. While conducting the experiments if one parameter was changed then the other property of the specimen was also changed.

Shariful et al. ${ }^{1}$ reported that the shrinkage properties of cotton spandex woven fabrics were mainly dependent on the heat setting temperature. If the heat setting temperature was more the specimen exposed less shrinkage value and vice versa.

Shariful et al. ${ }^{2}$ reported that the optimal strength of cotton spandex woven fabrics was attained with appropriate heat setting temperature. Overheat could damage the strength and other elastic performances of fabrics.

Shariful et al. ${ }^{3}$ also experimented that the elastic performances of cotton spandex woven fabrics were controlled with appropriate temperature treatment. Temperature application could control the elastic limit of the specimen.
Shariful et al. ${ }^{4}$ proved that the acoustic performances of needle punched nonwoven were improved with the application of sustainable natural fibers which were biodegradable and ecofriendly.

Shariful et al. ${ }^{5}$ also explained that the strength properties of cotton polyester blended woven fabrics were dependent on the polyester content percentage of the fabrics. Strength could improve if the polyester content percent was improved.

Shariful et al. ${ }^{6}$ also reported that there persist a relationship in between the strength and the polyester content percentage of the cotton polyester blended woven fabrics. If the polyester content percentage is increased the strength percentage is increased and vice versa.

Zhang et al. ${ }^{7}$ reported that the breathability of cotton fabrics were improved with the improvement of the cotton portion in the fabrics. As the natural cotton is breathable, biodegradable and absorbent and can easily contain moisture.

Ramzan et $a .^{8}$ also experimented that the effects of physical, mechanical and colorimetric properties of cotton woven fabrics were dependent on the cotton staple fibers. If the staple length was more the yarns exposed higher strength and vice versa.

Nagarajan et al. ${ }^{9}$ reported that the quality characteristics of bamboo cotton blended yarns of rotor and ring spun yarns was largely dependent on the staple length and hairiness index of the fibers.

Irshad et al. ${ }^{10}$ proved that the fabrics made up with short staple fibers caused hairy effect on fabrics. Fibers of long staple caused less hairy effect on fabric surface. 
Bai et al. ${ }^{11}$ proved that the effect of ply twist factor on hairiness and unevenness of two plied cotton yarns made of different spinning technologies were dependent on the staple fibers of cotton. Higher staple length with higher twist exposed more strength.

Cao et al. ${ }^{12}$ reported that the optimization of spinning parameters could influence the hairiness properties of polyester cotton vortex yarn.

Liang et al. ${ }^{13}$ proved that the increase of strength, toughness and electrical conductivity of twist-spun carbon nanotube yarns depends on the $\pi$ bridging of the carbon based.

Putra et al ${ }^{14}$ reported that the theoretical modeling for predicting yarn angle on OE yarn influenced by the fibre movement on torus coordinate based on classical mechanics approach.

Jiang et al. ${ }^{15}$ experimented that the shape of the garments is changed due to over shrinkage problem. If shrinkage problem is not controlled properly at fabric processing stage, it significantly affects the garments while manufacturing.

Shao et al. ${ }^{16}$ proved that the shrinkage problem is occurred in fabric due to getting uneven tension either from weaving loom or from wet processing operations.

Lim et al ${ }^{17}$ reported that while processing the fabrics at wet zone from pretreatment to finishing, the problem of shrinkage may be occurred due to uneven tension either by the mercerizing operation or by the stenter chain.

Shariful et al. ${ }^{18}$ conducted some experimental analysis of woven fabrics. They reported that fibers, yarns and fabrics have so many properties and these all properties are directly or indirectly modify the shrinkage properties of fabrics.

Table I Cotton polyester spandex denim fabrics of different fiber content

\section{Materials and method}

\section{Materials used}

Cotton polyester spandex denim fabric is the fundamental raw materials for this research. Cotton polyester blend along with spandex fibers were used in this research for conducting the required tests. Different types of cotton polyester spandex denim fabrics of different fiber content were used in this research as shown in Table 1. Finished raw denim fabrics were collected from fabric mills and the necessary shrinkages tests were carried out. Fabric of color blue indigo and Sulphur black were used in this research.

In the Table 1, for the given construction in serial no $A$, $16 \times(12+20 \mathrm{D}) / 115 \times 62$ shows a construction of a cotton polyester spandex denim fabric. Cotton polyester blending percentage is $65 \%$ cotton and $32 \%$ polyester. Along with this $3 \%$ spandex is inserted in weft way to provide elasticity. Here, warp yarn count is $16 \mathrm{Ne}$, which is a non-stretched yarn made up of cotton polyester blend, but weft yarn count is $(12 \mathrm{Ne}+20 \mathrm{D})$ that means, spandex of $20 \mathrm{D}$ denier is used in the core of $12 \mathrm{Ne}$ count in weft way to make it stretched yarn. Thread density in ends per inch is 115 and picks per inch is 62 . Two other constructions in serial no $\mathrm{B}$ and $\mathrm{C}$ show the same physiognomies. It is clear that percentage of spandex content depends on the value of Denier of spandex. The more the denier value is, the more the percentage of spandex content is. It is seen that $3 \%$ spandex content is achieved from the yarn of $(12+20 \mathrm{D}), 7 \%$ spandex content is achieved from the yarn of $(12+40 \mathrm{D})$ and $9 \%$ spandex content is achieved from the yarn of $(12+70 \mathrm{D})$.

\section{Method used}

Shrinkage tests were carried out in accordance with the test method AATCC (American Association of Textile Chemists and Colorists) 135. ${ }^{19}$

\begin{tabular}{|c|c|c|c|c|c|c|}
\hline S.N & Composition & Construction & Weave & Color & Width (") & Weight $\left(g / \mathrm{m}^{2}\right)$ \\
\hline A & $\begin{array}{l}65 \% \text { Cotton, } 32 \% \text { Polyester } \\
\text { and 3\% Spandex }\end{array}$ & $16 \times(12+20 D) / / 15 \times 62$ & $\begin{array}{l}\text { 3/I Right } \\
\text { Hand Twill }\end{array}$ & Blue Indigo & 55 & 306 \\
\hline B & $\begin{array}{l}75 \% \text { Cotton, } 18 \% \text { Polyester } \\
\text { and } 7 \% \text { Spandex }\end{array}$ & $16 \times(12+40 D) / 115 \times 62$ & $\begin{array}{l}\text { 3/I Right } \\
\text { Hand Twill }\end{array}$ & Sulphur Black & 55 & 306 \\
\hline C & $\begin{array}{l}85 \% \text { Cotton, } 6 \% \text { Polyester } \\
\text { and } 9 \% \text { Spandex }\end{array}$ & $16 \times(12+70 D) / / 15 \times 62$ & $\begin{array}{l}\text { 3/I Right } \\
\text { Hand Twill }\end{array}$ & Blue Indigo & 55 & 306 \\
\hline
\end{tabular}

\section{Machine used}

Shrinkage is the procedure where a fabric becomes smaller than its original size habitually over the practice of laundry. Dimensional stability to washing or shrinkage of a fabric was measured after 3 washes in accordance with "AATCC Test Method 135" with the facilities of a "TESTEX shrinkage washer TF176". Figure 1 shows a shrinkage testing machine. ${ }^{20}$

\section{Preparation of the samples}

Three types of cotton polyester spandex denim fabrics of different spandex content were collected from fabric mill just after finishing process is done for shrinkage testing as mentioned in Table 1. Using a measurement tape, sample size like "20-inch $\times 20$-inch" was cut but "18-inch $\times 18$-inch" was marked with permanent marker to prepare the fabric for shrinkage testing. Interlock stitching was given at the edge of the fabric before washing so that the yarns from the fabric could not be removed during heavy washing. This test method is applied to measure the dimensional changes of fabrics when subjected to home laundering. At first detergent washing was done for 90 minutes with $60^{\circ} \mathrm{C}$ temperatures. Secondly, the sample was rinse washed with room temperature for 45 minute. In rinse wash, fabric was just simply washed with normal clean water. Thirdly, extraction for 15 minutes and finally drying was done for 5 minute. After home laundry, shrinkage was measured with equation 1 in agreement with the AATCC Test Method 135 and shown the consequences in Figure 2, Figure 3, Figure 4 and in Figure 5. Figure 2 shows the arranged denim sample for shrinkage measurement. 
Shrinkage $\%=\left\{\frac{(\text { After Wash Length }- \text { Before Wash Length })}{\text { Before Wash Length }}\right\} 100$

Equation 1 Shrinkage measuring formula

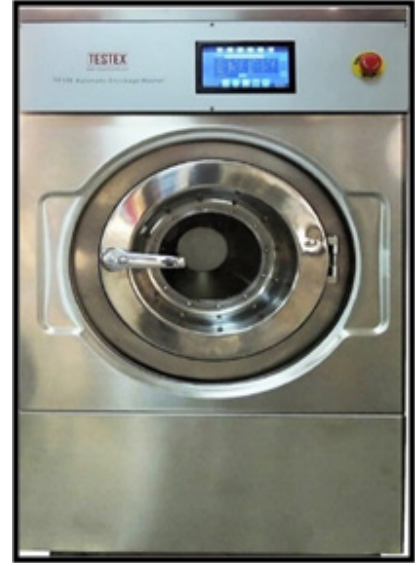

Figure I TESTEX Shrinkage Washer TFI76, China, 2014

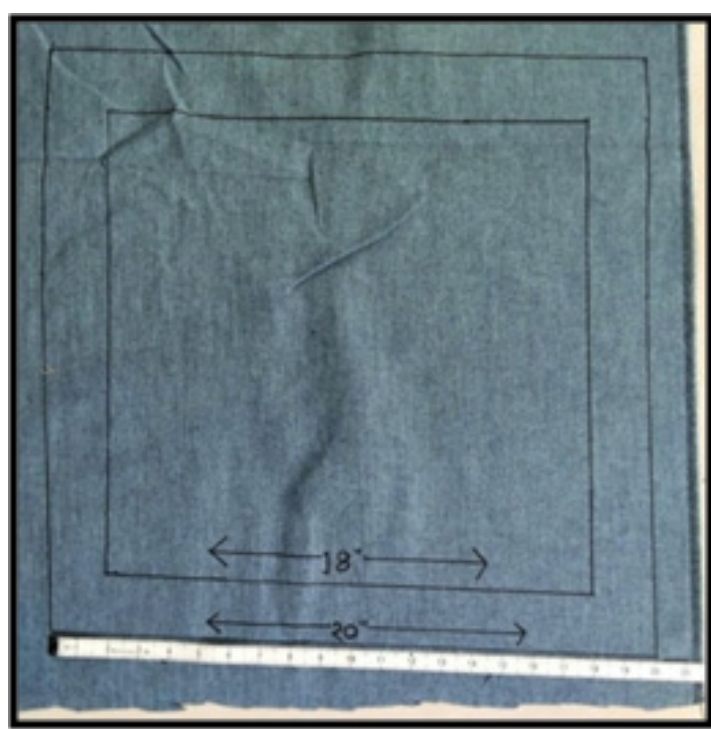

Figure 2 Sample Preparation for shrinkage testing.

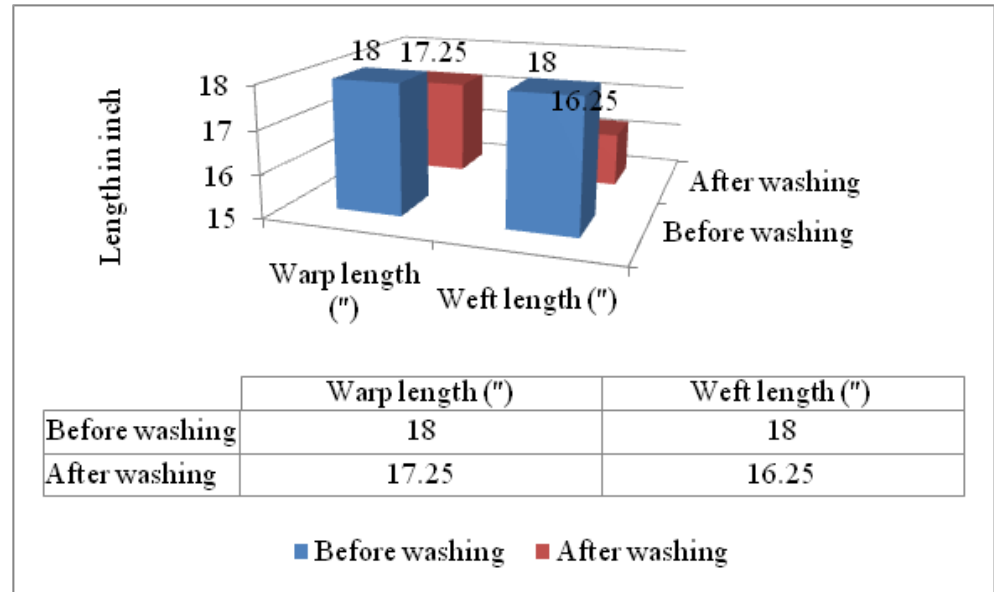

Figure 3 Warp and weft length of before and after washing of sample type A.

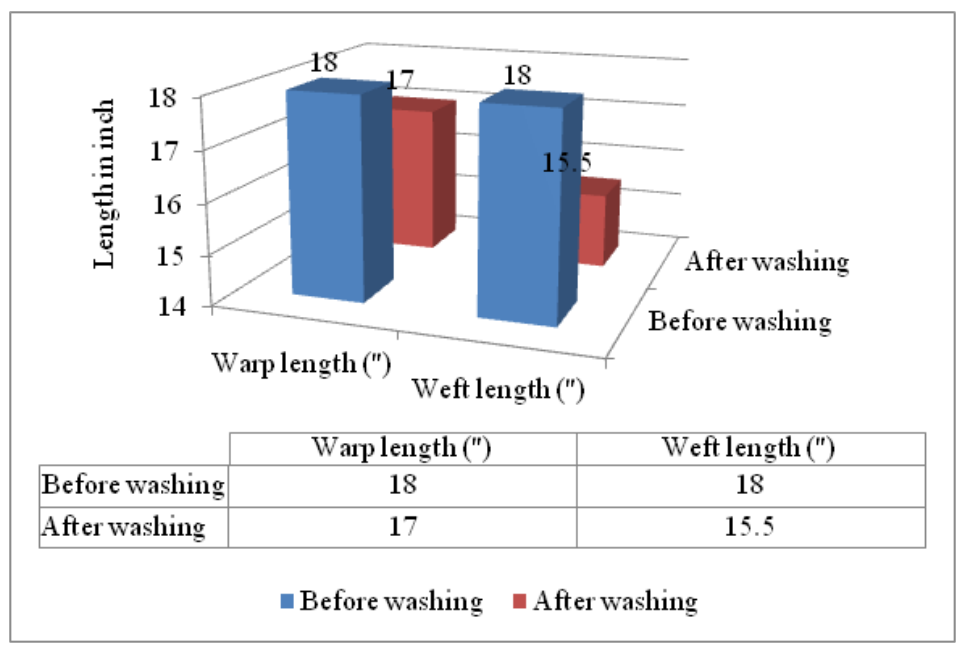

Figure 4 Warp and weft length of before and after washing of sample type B. 


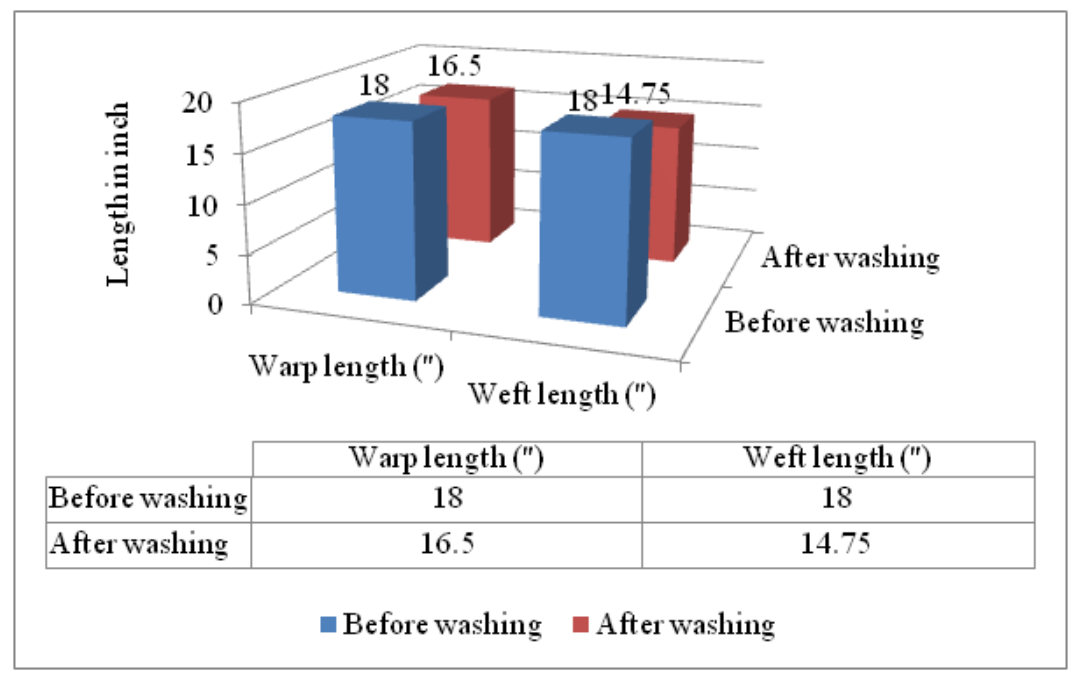

Figure 5 Warp and weft length of before and after washing of sample type C.

\section{The experimentation}

\section{Experiment A}

A specimen was taken that had a composition of $65 \%$ Cotton 32\% Polyester and 3\% Spandex with the construction of $16 \times(12+20 \mathrm{D}) / 115 \times 62$, had a width of 55 inches, weight of $306 \mathrm{~g} / \mathrm{m}^{2}$ and had a weave of $3 / 1$ right hand twill for doing the shrinkage tests. Color of the fabric was blue indigo. Measurement of the length of the samples before and after washing was taken in both warp and weft way and found the consequences shown in Figure 3. It is seen from the Figure 3 that, before washing the warp length was 18 " but after washing the warp length was 17.25 " and before washing the weft length was 18 " but after washing the weft length was 16.25 ".

\section{Experiment B}

A specimen was taken that had a composition of $75 \%$ Cotton $18 \%$ Polyester and $7 \%$ Spandex with the construction of $16 \times(12+40 \mathrm{D}) / 115 \times 62$, had a width of 55 inches, weight of $306 \mathrm{~g} / \mathrm{m}^{2}$ and had a weave of 3/1 right hand twill for doing the shrinkage tests. Color of the fabric was Sulphur black. Measurement of the length of the samples before and after washing was taken in both warp and weft way and found the consequences shown in Figure 4. It is seen from the figure 4 that before washing the warp length was 18 " but after washing the warp length was 17" and before washing the weft length was 18 " but after washing the weft length was 15.50 ".

\section{Experiment C}

A specimen was taken that had a composition of $85 \%$ Cotton $6 \%$ Polyester and 9\% Spandex with the construction of $16 \times(12+70 \mathrm{D}) /$ $115 \times 62$ had a width of 55 inches, weight of $306 \mathrm{~g} / \mathrm{m}^{2}$ and had a weave of $3 / 1$ right hand twill for doing the shrinkage tests. Color of the sample was blue indigo. Measurement of the length of the samples before and after washing was taken in both warp and weft way and found the consequences shown in Figure 5. It is seen from the Figure 5 that before washing the warp length was 18 " but after washing the warp length was 16.50 " and before washing the weft length was 18 " but after washing the weft length was 14.75 ".

\section{Result and discussion}

Experiments were carried out using three different nomenclatures mentioned in Table 1 with different fiber content and found the consequences of shrinkage shown in Figure 6, Figure 7 and in Figure 8. Using equation 1 and data obtained from Figure 3, Figure 4 and Figure 5 shrinkage values were achieved.

\section{Results of experiment A}

It is seen from the below Figure 6 that, the fabric containing $65 \%$ Cotton $32 \%$ Polyester and 3\% Spandex exposed the shrinkage values for warp $-4.16 \%$ and for weft $-9.72 \%$. In this type of sample $3 \%$ spandex content with $(12+20 \mathrm{D})$ shows less shrinkage values compared to the following samples in this research.

Cotton fibers and spandex shrink in water. Manmade elastic fiber shrinks more when it comes in contact of hot water. After laundry in hot water spandex and cotton shrinks more. On the other hand, polyester does not have any swelling property and it does not absorb water. Thereby it is not influenced due to laundering.

\section{Results of experiment B}

It is seen from the below Figure 7 that, the fabric containing $75 \%$ Cotton $18 \%$ Polyester and $7 \%$ Spandex exposed the shrinkage values for warp $-5.55 \%$ and for weft $-13.88 \%$. In this type of sample $7 \%$ spandex content with $(12+40 \mathrm{D})$ shows comparatively more shrinkage values than $3 \%$ spandex content with $(12+20 \mathrm{D})$.

Higher denier with higher spandex content shows more shrinkage values since it shrinks more while laundering. $75 \%$ cotton shrinks more in water compared to $65 \%$ cotton. Besides, $18 \%$ polyester shows more shrinkage values compared to $32 \%$ polyester. Cotton fibers and spandex shrink in water. Manmade elastic fiber shrinks more when it comes in contact of hot water. After laundry in hot water spandex and cotton shrinks more. On the other hand polyester does not have any swelling property and it does not absorb water. Thereby it is not influenced due to laundering. 


\section{Results of experiment C}

It is seen from the below figure 8 that, the fabric containing $85 \%$ Cotton 6\% Polyester and 9\% Spandex exposed the shrinkage values for warp $-8.33 \%$ and for weft $-18.05 \%$. In this type of sample $9 \%$ spandex content with $(12+70 \mathrm{D})$ shows comparatively more shrinkage values than $3 \%$ spandex content with $(12+20 \mathrm{D})$ and $7 \%$ spandex content with $(12+40 \mathrm{D})$.
Higher denier with higher spandex content shows more shrinkage values since it shrinks more while laundering. $85 \%$ cotton shrinks more in water compared to $75 \%$ cotton and $65 \%$ cotton. Besides, $6 \%$ polyester shows more shrinkage values compared to $32 \%$ polyester and $18 \%$ polyester content. Manmade elastic fiber shrinks more when it comes in contact of hot water. After laundry in hot water spandex and cotton shrinks more. On the other hand polyester does not have any swelling property and it does not absorb water. Thereby it is not influenced due to laundering.

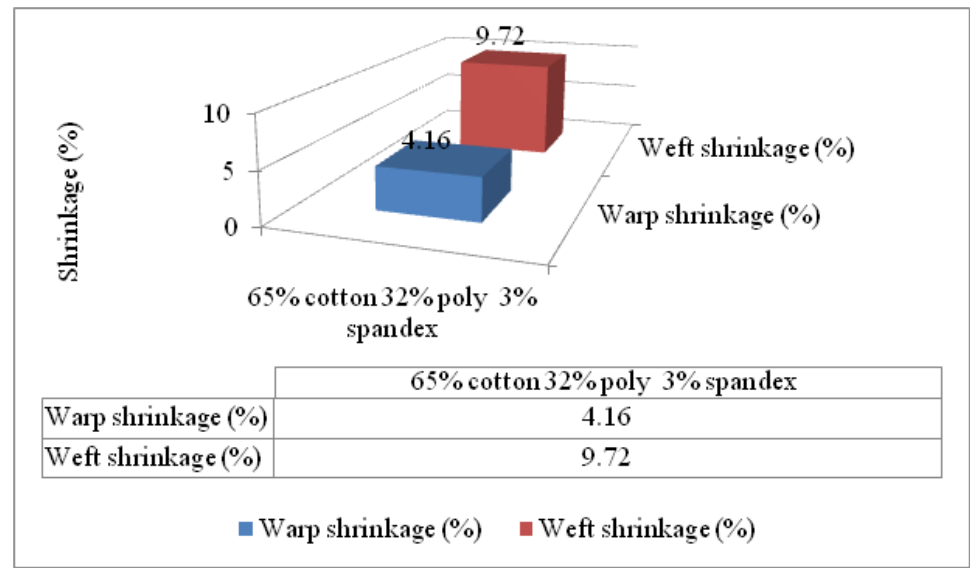

Figure 6 Warp and weft shrinkage of $65 \%$ cotton $32 \%$ polyester and $3 \%$ spandex.

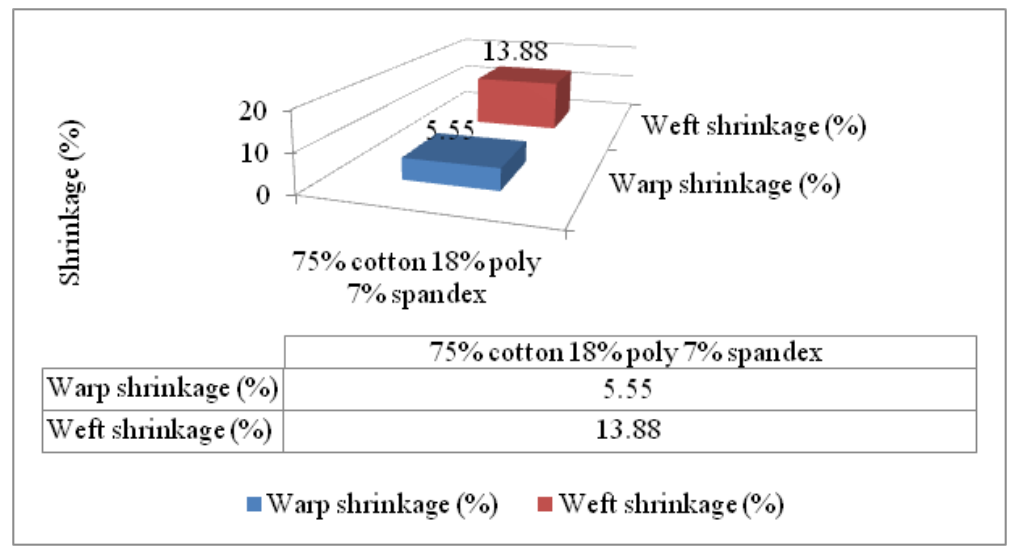

Figure 7 Warp and weft shrinkage of $75 \%$ cotton $18 \%$ polyester and $7 \%$ spandex.

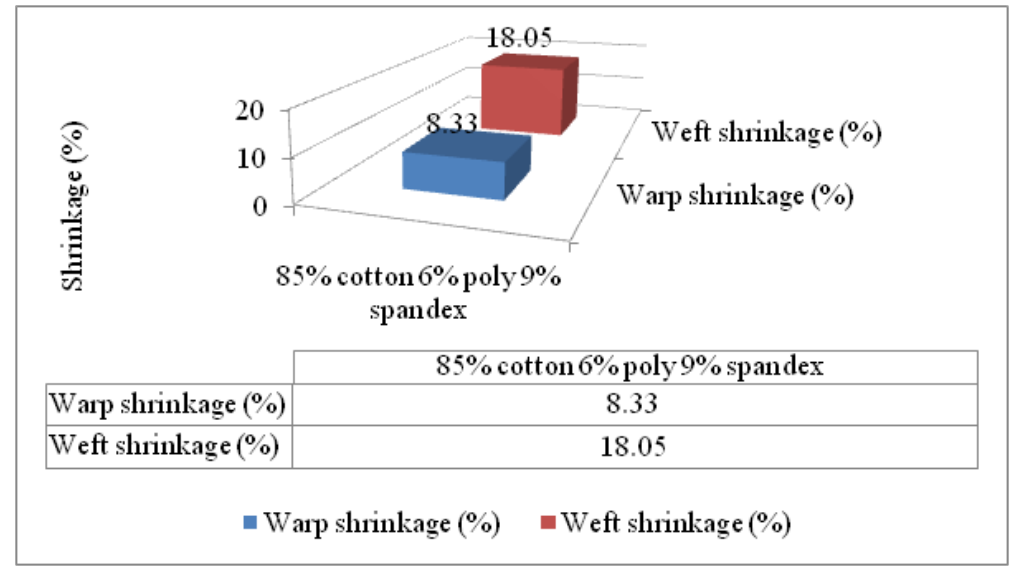

Figure 8 Warp and weft shrinkage of $85 \%$ cotton $6 \%$ polyester and $9 \%$ spandex. 


\section{Discussion}

It is seen from the Figure 6, Figure 7 and Figure 8 that shrinkage values were more for the fabrics of higher spandex content with higher spandex Denier. It was also noticed that, shrinkage values were more for the fabrics of higher cotton content but shrinkage values were less for the fabric of higher polyester content. The reason is that, cotton fabric has a tendency to shrink when it comes in contact to water. Cotton fibers shrink in water because they absorb water. When cotton absorbs water, they swell up, increase the width and there is a shortage of length, therefore shrinkage occurs. This incident happens more in hot water. Spandex fibers shrink more when temperature will be applied either in hot water or in steam compartment. Higher spandex content with higher denier values help the cotton fibers to shrink more then it's original state when submerged in water, that's why higher spandex content with cotton fibers give more shrinkage values. Therefore, the more the spandex content is, the more the shrinkage values are. On the other hand, manmade polyester does not swell in water and it does not shrink in contact of water. So, the more the polyester content is, the less the shrinkage percentage is.

\section{Conclusion}

In this research it was seen that the fiber composition had direct consequences on the shrinkage properties of cotton polyester spandex denim fabrics. Cotton fibers shrink in water due to its swelling property in water. So, the more the cotton fibers in a blend are, the more the shrinkage values are. Likewise, spandex fibers shrink more when it comes in contact of either in hot water or in steam compartment. When hot water, steam or hot air comes in contact of spandex content, then it shrinks due to its rubber like elasticity. Manmade polyester fibers never shrink in water as it does not have any swelling property in wet condition. If the polyester content is more in the blend, the shrinkage property will be less. Shrinkage properties may differ if the machine setting of shrinkage tester, time or temperature is changed. It is proved in the research that the fabric of $85 \%$ Cotton, $6 \%$ Polyester and $9 \%$ Spandex content shows most shrinkage values compared with other samples in this research due to the higher spandex content with higher denier values. Trials were carried out in textile mills with denim fabrics of various fiber content of cotton, polyester and spandex to study the irregular shrinkage properties found in elastic denim fabric. This research is practiced based and the findings are beneficial to the personnel involved in textile industries who are responsible for the manufacture of cotton spandex woven fabrics. This research opened several ways for the scholars to further study in this field.

\section{Acknowledgments}

None.

\section{Conflicts of interest}

The authors declare that they have no competing interests.

\section{References}

1. Islam S, Alam SMM, Akter S. The consequences of temperature on the shrinkage properties of cotton spandex woven fabrics. Journal of Textiles and Polymers. 2018;7(1):2-7.

2. Islam S. Attaining optimum strength of cotton-spandex woven fabric by apposite heat-setting temperature. Journal of The Institution of Engineers (India): Series C. 2018;8(35):1-6.

3. Islam S, Alam SMM, Akter S. Identifying a suitable heat setting temperature to optimize the elastic performances of cotton spandex woven fabric. Research Journal of Textile and Apparel, Emerald Publishing Group. 2018;22(3):260-270.

4. Islam S, Mominul Alam SM. Investigation of the acoustic properties of needle punched nonwoven produced of blend with sustainable fibers. International Journal of Clothing Science and Technology. 2018;30(3):444-458.

5. Islam Shariful, Yasmin Jarin, Alam Syed, et al. Identifying the strength properties of cotton polyester blended woven fabrics of different fiber content. Research Journal of Material Sciences. 2019;7(2):1-6.

6. Islam Shariful, Ahmed Shaharia, Arifuzzaman, et al. Relationship in between strength and polyester content percentage of cotton polyester blended woven fabrics. International Journal of Clothing Science. 2019;6(1):1-6.

7. Zhang Y, Tian W, Liu L, et al. Eco-friendly flame retardant and electromagnetic interference shielding cotton fabrics with multi-layered coatings. Chemical Engineering Journal. 2019;372:1077-1090.

8. Ramzan MB, Rasheed A, Ali Z, et al. Impact of wash types and stitching parameters on shrinkage of knitwear made from pique fabric. International Journal of Clothing Science and Technology. 2019;31(2):232-242.

9. Nagarajan G, Ramachandran DT, Boobalan S. An analysis of quality characteristics of bamboo/cotton blended yarns of rotor and ring spun. International Research Journal of Science and Technology. 2019;1(1):31-34.

10. Irshad F, Ahmed T, Farooq A, et al. Effect of cross linker treatment on dimensional and mechanical properties of knitted fabrics. Pakistan Journal of Scientific \& Industrial Research Series A: Physical Sciences. 2019;62(1):48-51.

11. Bai S, Xu R, Wang W, et al. Dual-response of temperature and humidity asymmetrical cotton fabric prepared based on thiol-ene click chemistry. Colloids and Surfaces A: Physicochemical and Engineering Aspects. 2019;567:104-111.

12. Cao H, Zulifqar A, Hua T, et al. Bi-stretch auxetic woven fabrics based on foldable geometry. Textile Research Journal. 2019;89(13):2694-2712.

13. Liang X, Gao Y, Duan J, et al. Enhancing the strength, toughness, and electrical conductivity of twist-spun carbon nanotube yarns by $\pi$ bridging. Carbon. 2019;150:268-274.

14. Putra VGV, Rosyid MF, Maruto G. New theoretical modeling for predicting yarn angle on $\mathrm{OE}$ yarn influenced by fibre movement on torus coordinate based on classical mechanics approach. Indian Journal of Fibre \& Textile Research (IJFTR). 2017;42(3):359-363.

15. Jiang $\mathrm{Z}$, Yu C, Yang J, et al. Estimation of yarn strength based on critical slipping length and fiber length distribution. Textile Research Journal. 2019;89(2):182-194.

16. Shao R, Cheng L, Xue W, et al. Theoretical study of the effects on yarn strength in a modified ring spinning system. Textile Research Journal. 2019 .

17. Lim YW, Kwon OE, Kang SM, et al. Built $\square$ in haze glass $\square$ fabric reinforced siloxane hybrid film for efficient organic light $\square$ emitting diodes (OLEDs). Advanced Functional Materials. 2018;28(33):1802944.

18. Islam S, Chowdhury S, Akter S. The experiential analysis of woven fabric for reproduction. Journal of Textile Science and Technology. 2018;4(1):18.

19. The shrinkage testing method AATCC 135. Standard test methods for shrinkage of cotton farbic blended with different fiber content. North Carolina, USA: AATCC International, Research Triangle Park; 2013;1:31-35.

20. The Shrinkage Testing Machine. TESTEX Shrinkage Washer TF176, 2014. NO. 3, Daliantang Industrial Dt, Wan Jiang, Dongguan, 523000, P.R. China; 2014. 